\title{
Reliability Improvement of Power Distribution Systems using Advanced Distribution Automation
}

\author{
M. R. Elkadeem, M. A. Alaam and Ahmed M. Azmy \\ Elec. Power and Machines Eng. Depart., Faculty of Eng., Tanta University, Tanta, Egypt \\ mohammad.elkadim@f-eng.tanta.edu.eg,mhmd.aboelazem@f- \\ eng.tanta.edu.eg, azmy@f-eng.tanta.edu.eg
}

\begin{abstract}
Towards the complete vision of smarter distribution grid, advanced distribution automation system (ADAS) is one of the major players in this area. In this scope, this paper introduces a generic strategy for cost-effective implementation and evaluation of ADAS. Along with the same line, fault location, isolation and service restoration (FLISR) is one of the most beneficial and desirable applications of ADAS for selfhealing and reliability improvement. Therefore, a localcentralized-based FLISR (LC-FLISR) architecture is implemented on a real, urban, underground medium voltage distribution network. For the investigated network, the complete procedure and structure of the LC-FLISR are presented. Finally, the level of reliability improvement and customers' satisfaction enhancement are evaluated. The results are presented in the form of a comparative study between the proposed automated and non-automated distribution networks. The results show that the automated network with proposed ADAS has a considerable benefit through a significant reduction in reliability indices. In addition, it has remarkable benefits observed from increasing customers' satisfaction and reducing penalties from industry regulators.
\end{abstract}

Keywords - Advanced distribution automation system, Fault location, isolation and service restoration, Selfhealing grid, Relaibilty assessment study.

\section{INTRODUCTION}

In the contemporary world, the electrical distribution system is the backbone of the smart grid environment. However, most of customer outages are a result of the distribution networks, where $80 \%$ of customer interruptions attribute to faults and component failure at the feeder level [1]. Conventional distribution systems do not involve much automation i.e. rely mainly on manual operations, and do not have any communication or information exchange i.e. blind system. During system events, the absence of monitoring and lack of information with increasing failure rate would dramatically increase the duration and number of customers affected by specific outage. The technical impact will appear in decreasing the reliability level of the network and the quality of supply. The significant economic impact of this would be observed in increasing the outages cost of utility and their customers. For these reasons, improving system reliability and power quality becomes an important issue.

Keeping the above problems in mind, distribution automation (DA) is the key answer to many challenges facing electrical distribution networks. Referring to electrical power research institute (EPRI), advanced distribution automation system (ADAS) has been defined as "a fully controllable and flexible distribution system that will facilities the exchange of electrical energy and information between participants and system components" [2], [3]. The expected results of applying DA on medium voltage (MV) distribution networks include, for example, reliable, resilience, selfhealing, fully controllable and efficient distribution system for energy delivery to end users in customers' domain. Much of efforts that depend upon modernizing the distribution network are accomplished and many utilities around the world started distribution automation projects [4]-[9]. ADAS combines different applications that have been discussed in [10] and summarized as follows:

- Fault location, isolation, and service restoration

- Volt/Var control and optimization

- Distributed generation resources management

- Adaptive distribution feeder protection coordination

- Optimal feeder reconfiguration

- Automatic meter reading

- Demand side management 
Fault location, isolation and service restoration (FLISR) is defined as the most beneficial, attractive application among all applications of ADAS in order to getting self-healing grid, enhancing the reliability level of distribution networks, reducing operation and maintenance cost and increasing customers satisfaction. This is achieved by means of reducing the outage time from several hours to few minutes [11]. There are different architectures for FLISR implementation based on where the decision is made: fully-centralized (FC-FLISR) [6],[7], fully-decentralized (FD-FLISR) [8],[9] and local-centralized (LC-FLISR) architectures. In fully centralized architecture, the FLISR algorithms are implemented in DMS/SCADA system located at the control centre (CC) in so far area. In the decentralized architecture, the FLISR uses an intelligent local controller, integrated with the multiple switches distributed along feeder nodes.

The scope of this paper is to evaluate the effective utilization of ADAS within power distribution systems. In the context of ADAS implementation, to avoid the huge investment and maximize the benefits towards a complete vision for the smart distribution grid, a generic strategy for ADAS is developed in this paper. Also, a local-centralized architecture is presented as the most appropriate architecture for FLISR implementation within the investigated distribution network. The reasons and encouragements for this choice are discussed in the next sections. In addition, the structure and procedure of FLISR system are presented. Finally. The reliability assessment study is performed using analytical technique. The results are presented in the form of a comparative study between the proposed automated and non-automated distribution networks. The results show that the automated network with proposed ADAS is able to achieve a significant and considerable improvement in reliability level of the distribution network.

\section{DESCRIPTION AND OPERATION OF THE DISTRIBUTION NETWORK UNDER STUDY}

\section{A. Description of the investigated distribution network}

To prove the validity of the proposed study, it will be performed on a real distribution network as a case study. This distribution network is an actual, urban, underground, $11 \mathrm{kV}$ rated distribution network with open-ring configuration. As demonstrated in Fig. 1, the investigated network consists of an $11 \mathrm{kV}$ distribution substation fed from a $22 \mathrm{kV}$ distribution system, which has a 750 MVA short circuit capacity. This distribution substation supplies four outgoing feeders. Each is protected by and intelligent electronic devices (IED) at the beginning of the feeder. The lengths of these feeders are $5.485 \mathrm{~km}, 1.750 \mathrm{~km}, 3.725 \mathrm{~km}$, and 6.725 $\mathrm{km}$, respectively. The distribution network contains thirty-five $11 \mathrm{kV}$ compact secondary substations (CSSs), which are installed over the four feeders. Each CSS is a fully equipped package substation divided into three main compartments: incoming MV compartment, transformer (XFMR) compartment and low voltage feeder panel (LVFP) compartment as illustrated in

Fig. 2.

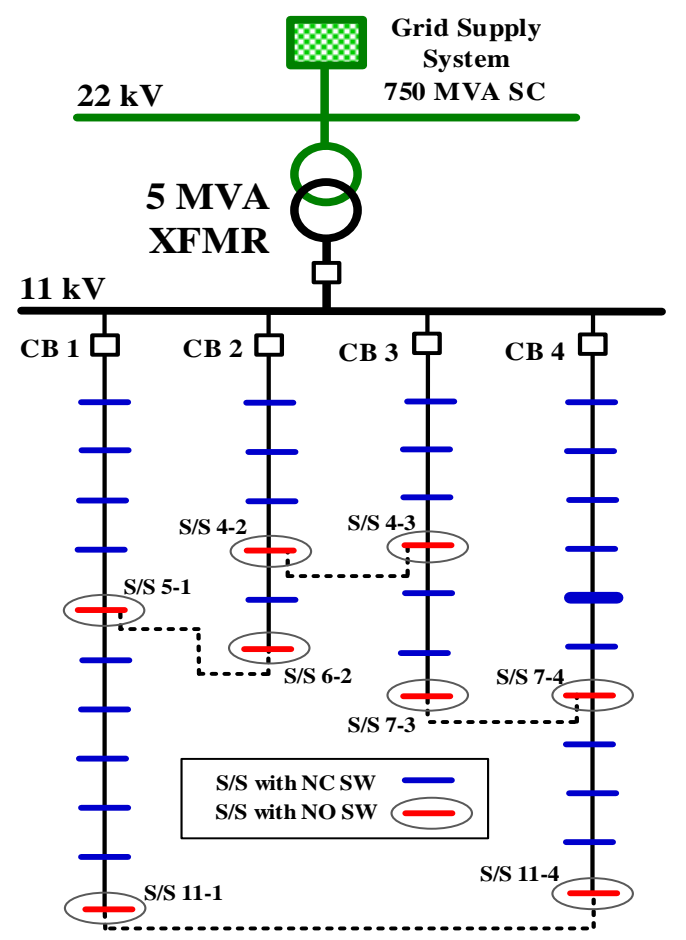

Fig .1. $11 \mathrm{kV}$ Underground distribution network

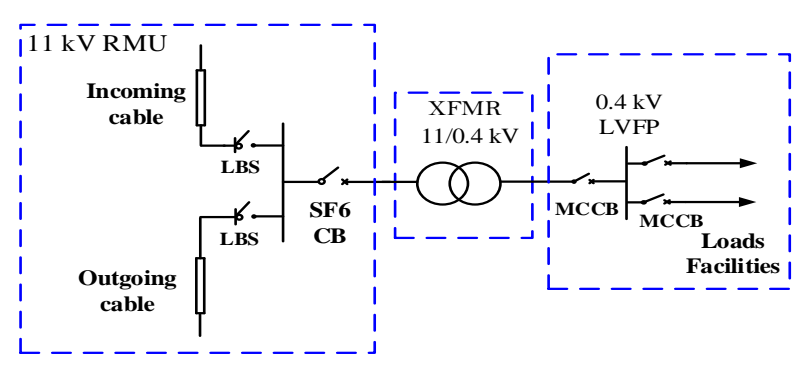

Fig .2. Compact secondary substation compartments 


\section{B. Operation mode of the investigated distribution network}

The existing MV distribution network is a conventional network and have not involved much automation i.e. rely mainly on manual operations. Traditional FLISR still exhibits long time and stressful efforts as follows:

- The fault is detected via the outage reports received from the outage customers.

- Afterwards, investigation process for fault localization to determine the faulty section is started. This process necessitates the patrolling of the entire network.

- For successful isolation of the faulty section, manual-switching actions are completely accomplished by human intervention.

- Finally, other manual switching actions are executed to restore the service to healthy customers.

Briefly, FLISR is performed manually via human intervention and hence, the network operator is facing some of technical and financial issues that are defined and represented by increasing the outages time and outages costs, especially with the presence of the underground cables spread over long distances. Consequently, it is suffered from low level of service reliability and low customers' satisfaction.

\section{THE PROPSED ADVANCED DISTRIBUTION AUTOMATION SYSTEM}

Motivated by the earlier mentioned issues in the previous section, ADAS is an imperative solution in order to strengthen the operation of MV distribution networks. This following section attempt to discuss some issues related to ADAS implementation from different points of view, e.g. ADAS strategy of implementation, selection of the most appropriate architecture. Finally, the structure and requirements of the proposed automation system is introduced.

\section{A. Implemented strategy of ADAS}

Although it may be not possible to describe a successful ADAS strategy, rapid deployments worldwide have thought some lessons [11], [12] that help to construct the proposed implemented strategy for the existing distribution network, as illustrated in Fig. 3. The target of the proposed ADAS strategy is to determine the main requirements of ADAS including selection of the most appropriate architecture or approach for ADAS, defining of automation degree and complete structure of ADAS. The detailed explanation of the proposed ADAS strategy will be presented as follows:

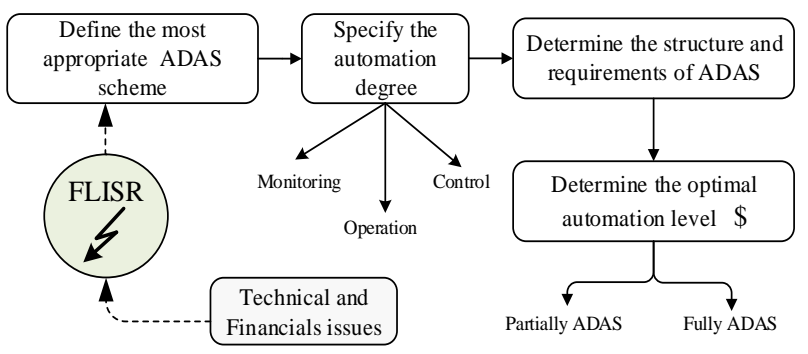

Fig .3. General Strategy for distribution automation implementation

The proposed strategy starts with selection of the most significant and beneficial application that is currently needed for the system. This application will be the first phase of the smart distribution network and will be defined according to the existing technical and financial issues facing the distribution network e.g. for the existing distribution network, which is presented as a case study, FLISR is defined. The second step is to define the most appropriate architecture for ADAS. The ADAS scheme is refer to where the decision is made. Three different scheme are introduced in section I. The selection criteria are based on the requirements of the defined application, the nature of the existing control strategy of the network, network size and configuration, as well as economic resources.

The next step is the specification of the automation degree. The automation degree is categorized into three categories; monitoring, control, and operation or any combination of them. This is based on the network operator target and requirements. After that, utility have to determine the copmlete structure and requirements of the ADAS. In general, the main structure and requirements of ADAS include: flexible electrical hardware facilities, modern communication technologies and intelligent software applications [11]. Finally, for for reasonable degree of improvement in system performance and economic justification and profitability, cost/benefit analysis is performed to determine the optimal automation level of the distribution network. The automation level of MV distribution network is considered to be percentage of 
automated points in the network. For underground networks, the automated points are the number of secondary substations $(\mathrm{S} / \mathrm{Ss})$ retrofitted with smart devices such as feeder remote terminal units and fault indicators.

\section{B. Proposed Local-centralized FLISR}

Nowadays, the great evolution of smart sensors, advanced communication technologies and utilization growing of SCADA/DMS, accelerates the transformation of the traditional distribution networks into automated systems. Following the proposed strategy for ADAS, FLISR application is defined as the most appropriate solution among all applications of ADAS to achieve self-healing grid and to improve the reliability level of distribution network. FLISR is implemented on the existing MV distribution network based on local-centralized architecture, called (LCFLISR). The information is acquired and analysed in primary substation. Then, the control decision is executed automatically on real network or after confirmed by the operator using the SCADA interface. The idea behind FLISR is to achieve the self-healing grid, where the service for all customers is restored automatically after few minutes ( $<5$ minutes) from fault from fault occurrence [11]. The main objectives of the LC-FLISR are illustrated in Fig. 4. So, As shown in Fig. 5, the proposed structure of ADAS consists of:

Therefore, this paper aims to present a simple and more efficient CBPWM technique to control three to five-phase matrix converters, which can be used to supply a five-phase induction motor drive system that delivers some advantageous features for industrial applications. The basic concept of the proposed technique is already published in [27]. Thanks to its simplicity, the proposed technique will be based on the indirect modulation of the $3 \times 5 \mathrm{MC}$ which control the converter as a double stage converter. Therefore, the carrier based PWM methods are applied for each stage independently. The proposed modulation will maximize the converter VTR by operating the converter in the overmodulation mode as well as in the linear modulation mode. It also controls the input power factor by controlling the input current displacement angle. The proposed modulation technique is verified using simulation and experimental results based on a laboratory prototype and the dSPACE-DS1104 controller platform and the results are compared by the existing SVM technique.
- Master unit controller (called MU) as the "decision support system" of the ADAS. The information is acquired and analysed by MU. Then, the control decision is executed automatically on real network or after confirmed by the operator using the SCADA interface

- Intelligent software applications (FLISR algorithms); as this paper focus on FLISR application. Thereby, there are two main intelligent algorithms are needed to be integrated with ADAS: "fault location" and "service restoration" algorithms.

- Modern communication technologies as the heart of ADAS. The core mission of the network communications is to provide information exchange facilities between field devices dispersed geographically along the distribution grid and master unit located at primary substation.

- Flexible electrical hardware facilities (field devices).

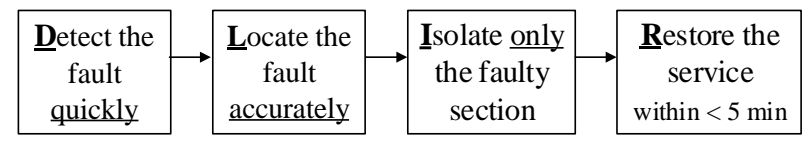

Fig .4. FLISR Objectives

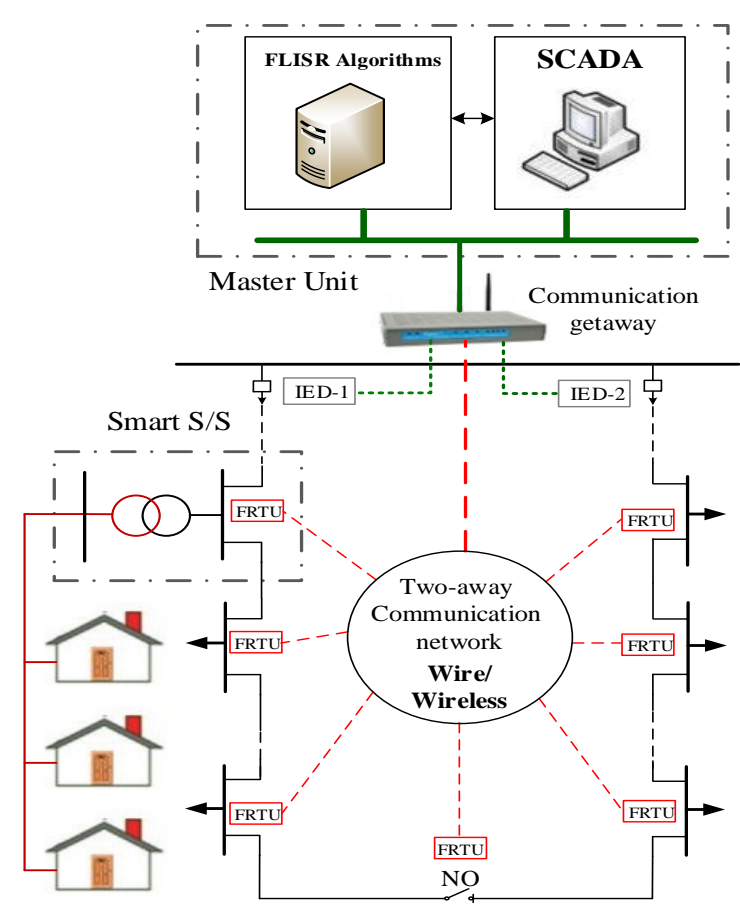

Fig .5. Over view of local-centralized ADAS structure 
Regardin to field devices, the secondary substations play a vital role in the evolution toward the self-healing grid. Therefore, the retrofitting of the existing traditional $\mathrm{S} / \mathrm{Ss}$ with smart equipment suitable for ADAS. A typical configuratuon of smart $S / S$ is presented in Fig. 6, where the figure is taken from [13]. However, the smart devices that are suitable for the propsed ADAS are only highlighed. So, the smart S/S is consists of: smart RMU equipped with motorized LBSs, motorized CB, fault indicators (Fls), voltage indicators (VIs), and uninterruptible power supply (UPS). Also, the smart S/S is supported with feeder remote terminal unit (FRTU) acts as a local controller for field devices. In addition, FRTU acts as a gateway between field devices and MU. From the literature [14], the communication between MU and FRTUs in this paper will take place through public GPRS communication based on IEC 60870-5-101 protocols as a cost-effective solution [5]. On the other hand, connection with field devices will be wired (serial RS232/RS-458) using conventional IEC 60870-1-104 or Modbus RTU protocols supported by field devices. On the way to realise a high level of reliability, reducing outage time and outage cost, increasing customer satisfaction, a fully automated system is implemented. So, all S/Ss would be retrofitted with smart equipment that are suitable for DAS.

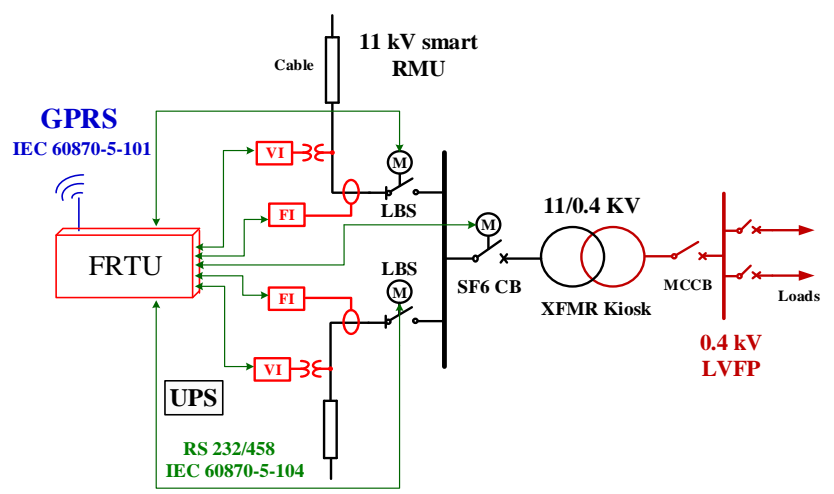

Fig.6. Typical configuration of smart S/S with ADAS equipment

\section{Proposed ADAS architecture:}

In this paper LC-FLISR architecture is selected as the most appropriate solution due to the following reasons:

The choice of a LC-FLISR architecture to be implemented in this paper is judged by the following reasons:
- Compared to fully centralized architecture, there is no need for huge investment that is required for FCFLISR scheme including SCADA/DMS hardware and software. The decision is taken from MU located at the primary substation within the same graphical area of the entire network. The results, the communication is over relatively short distances. Hence, high-speed data transfer is achieved and the service restoration time is lower. The significant economic impact of would be observed in decreasing the customer outages cost of utility and their customers.

- Compared to distributed architecture that has limited functionality, in LC-FLISR, the structure of constructed algorithm located in primary substation is easy to be modified and expanded to accommodate the upcoming extensions in the network. Also, because of the communication network between MU and RFTUs are already used in the system e.g. GPRS network, a few costs are required with adding more ADAS application such as volt/var control and Distributed generation resources management

\section{LC-FLISR procedur}

In this subsection, the steps of LC-FLISR procedure are discussed as follows:

- Firstly, with permanent fault occurrence the IEDs at the beginning of each feeder will detect and initiate the corrective action as quickly as possible for the corresponding feeder $\mathrm{CB}$.

- With the help of the FPIs installed in each smart $\mathrm{S} / \mathrm{Ss}$, the faulty section well-known between the last flashed $\mathrm{FI}$ and first unflashed FI.

- The next is to isolate only the faulty section. So, a control commands are automatically issued by MU in order to open the proper LBSs surrounding the fault.

- After the faulty section is isolated successfully, the LC- FLISR algorithms are triggered swiftly through SCADA inputs such as LBSs and FPIs statuses. With fault location capability integrated with the proposed ADAS system. The probable location of the faulty point will be estimated, [15] rather than determining the faulty section. Consequently, the fault damage inspection and repairing time and money can be saved. 
- Finally, the service can be restored again for the customers of remaining healthy sections by the help of the service restoration algorithm implemented in MU. the service restoration algorithm is responsible for generating a suitable restoration-switching plane to the proper LBSs in the system to achieve the goal [16]. As a result, it will improve the service reliability and increases the operational efficiency of the network.

\section{RELIABILITY ASSESSMENT STUDY}

\section{A. Reliability indices and technique}

In order to quantify and evaluate the benefit of ADAS implementation, reliability assessment study is performed. System reliability refers to the capability of the system to perform its specified task correctly for certain duration of time. The system reliability is represented using two terms "system adequacy" and system security". There are two categories of indices that used to define the reliability level of the distribution system; load point reliability indices and system reliability indices. The load point indices include; average failure rate, $\lambda \mathrm{i}(\mathrm{f} / \mathrm{yr})$, expected outage duration, ri (hr), and unavailability, Ui (hr/yr) for load point " $i$ " [17]. Also, the expected energy not supplied index at each load point, EENSi (MWhr/yr) can be used to assess the performance of the system. From theses load point indices, the system reliability indices such as SAIDI, SAIFI, CAIDI and EENS can be then calculated for the whole system [17]. Theses indices can be calculated according to references, [18], [19] as follows:

$$
\begin{aligned}
& \lambda_{i}=\lambda_{1}+\lambda_{2}+\cdots \lambda_{M}=(f / y r) \\
& U_{i}=\lambda_{1} r_{1}+\lambda_{2} r_{2}+\cdots \lambda_{M} r_{M}= \\
& \sum_{X=1}^{M} \lambda_{X} r_{X} \quad(h r / y r) \\
& r_{i}=\frac{U_{i}}{\lambda_{i}}=\frac{\sum_{x=1}^{M} \lambda_{X} r_{X}}{\sum_{X=1}^{M} \lambda_{X}} \quad \text { (hr) } \\
& E_{E N S_{i}}=P_{i} U_{i} \quad(M W h r / y r)
\end{aligned}
$$

$$
\begin{aligned}
& S A I D I=\frac{\sum_{i=1}^{N_{T}} U_{i} N_{i}}{N_{T}}(h r / y r) \\
& S A I F I==\frac{\sum_{i=1}^{N_{T}} \lambda_{i} N_{i}}{N_{T}}(\mathrm{int} / \mathrm{yr}) \\
& C A I D I=\frac{\text { SAIDI }}{\text { SAIFI }} \quad(\text { hr/int }) \\
& \text { EENS }=\sum_{i=1}^{N_{T}} P_{i} U_{i} \quad(M W h r / y r)
\end{aligned}
$$

where: $\lambda_{j}$ is failure rate of component $j(f / y r), M$ is the number of components that affect the load point " $\mathrm{i}$ ", rj "is" the restoration time of load point " $i$ " after failure of component " $\mathrm{x}$ " (hr), $\mathrm{P}_{\mathrm{i}}$ is the total demand at load point "i" and $\mathrm{N}_{T}$ is the total number of customers served (MW).

The reliability level of the distribution system depends mainly on the overall time of the FLISR procedure after fault event. This time is estimated according to the operating strategy non-automated and automated distribution network. In this paper, the reliability study is performed using analytical technique based on failure mode effect analysis (FMEA) [20]. The basic procedure used in the analytical technique is shown in Fig. 7.

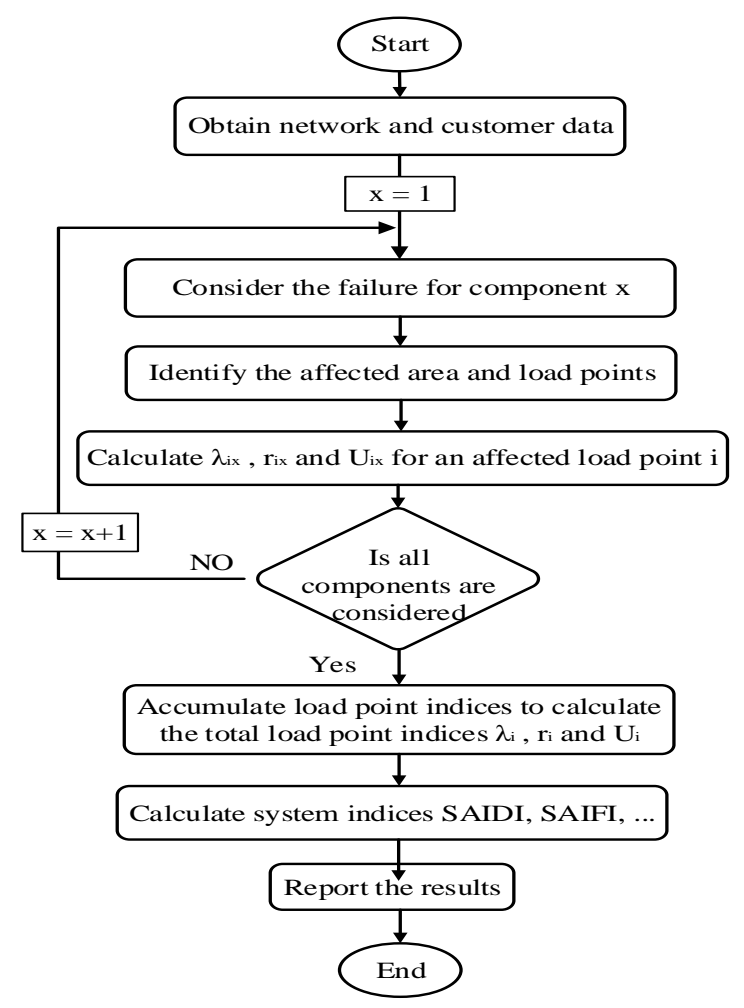

Fig. 7. Procedure of the analytical technique for reliability assessment 

http://dx.doi.org/10.21622/RESD.2017.03.1.024

\section{B. Simulation results}

The structure and configuration of the investigated 11 $\mathrm{kV}$ distribution network is presented in section II-A. The reliability indices of the distribution network are evaluated with two operating philosophy: nonautomated distribution network strategy $e$ and automated distribution network. Then, the results are compared in order to ensure the achievement of improving the reliability indices using ADAS. The network reliability data are taken from IEEE Standard 493-2007 (Golden book) [19], and given in Table. 1.

Table 1. NETWORK RELIABILITY DATA

\begin{tabular}{|c|c|c|c|c|}
\hline $\begin{array}{c}\text { Equipment } \\
\text { category }\end{array}$ & $\begin{array}{c}\text { Failure rate } \\
\text { (failure/year) }\end{array}$ & $\begin{array}{c}\text { Repair time } \\
\text { (h/failure) }\end{array}$ & $\begin{array}{c}\text { Unavailability } \\
\text { (h/year) }\end{array}$ & Comments \\
\hline LV CB & 0.0027 & 4 & 0.0108 & \\
\hline MV CB & 0.0036 & 2.1 & 0.0076 & \\
\hline LV Cable & 0.00141 & 10.5 & 0.0148 & 1000 circuit ft. \\
\hline MV Cable & 0.00613 & 26.5 & 0.1642 & 1000 circuit ft. \\
\hline Disc. Switch & 0.0061 & 3.6 & 0.022 & \\
\hline MV Transf. & 0.003 & 130 & 1.026 & $\begin{array}{c}\text { Replace by } \\
\text { spare }\end{array}$ \\
\hline LV SWGR & 0.0024 & 24 & 0.0576 & \\
\hline MV SWGR & 0.0102 & 26.8 & 0.2733 & \\
\hline
\end{tabular}

The reliability study is performed under the following assumption: normally, the distribution network operates in open-ring configuration, but some of the network feeders can be connected to each other through the normally open tie switch and the average restoration time for the healthy customers defined to be under 5 minutes with automated network and two hours with non-automated one. Also all the CBs and LBSs are assumed to be $100 \%$ reliable and all failures are statistically independent.

The load point reliability indices such as $\lambda, r, U$ and EENSI are calculated using FMEA method with the help ETAP $\AA 12.60$ software package and the results are presented in Table 2 for comparative study purpose between non-automated and automated network. In addition, the system reliability indices such as SAIDI, SAIFI, and EENS are also calculated for each of four feeders and whole system and given in Table 3. The results indicate clearly that the load point indices are improved significantly for all load points in the investigated distribution network by implementing the proposed ADAS. In addition, there are a clear reduction in system indices, i.e. SAIDI and EENS, for all system feeders and whole system. For the whole system, there is a reduction in both SAIDI and EENS by $34 \%$ and $32 \%$ respectively by using ADAS as shown in Fig. 8. This is observed through the reduction in reliability indices. So, this will directly lead to the improvement of the reliability level of the investigated distribution network, increase customer satisfaction, reducing system interruption cost for various load categories (residential, commercial, industrial and so on) by quick restoration of service and reducing penalties and achieve incentives offered by regulators.

Table 2. Results summary of load point indices for non-automated and automated distribution network

\begin{tabular}{|c|c|c|c|c|c|c|c|}
\hline \multirow[b]{2}{*}{ LP } & \multirow[b]{2}{*}{ Feeder } & \multicolumn{3}{|c|}{ Non-automated } & \multicolumn{3}{|c|}{ Automated } \\
\hline & & $\begin{array}{c}r \\
\text { (hr) }\end{array}$ & $\underset{(\mathrm{hr} / \mathrm{yr})}{\mathrm{U}}$ & $\begin{array}{c}\text { EENSI } \\
\text { (MWhr/yr) }\end{array}$ & $\begin{array}{c}\mathrm{r} \\
(\mathrm{hr})\end{array}$ & $\underset{(\mathrm{hr} / \mathrm{yr})}{\mathrm{U}}$ & $\begin{array}{c}\text { EENSI } \\
\text { (MWhr/yr) }\end{array}$ \\
\hline 1 & $\# 1$ & 4.744 & 1.108 & 0.148 & 3.029 & 0.707 & 0.094 \\
\hline 2 & $\# 1$ & 4.916 & 1.157 & 0.284 & 3.214 & 0.757 & 0.186 \\
\hline 3 & $\# 1$ & 4.826 & 1.131 & 0.303 & 3.117 & 0.731 & 0.196 \\
\hline 4 & $\# 1$ & 5.023 & 1.188 & 0.172 & 3.330 & 0.788 & 0.114 \\
\hline 5 & $\# 1$ & 4.934 & 1.162 & 0.081 & 3.234 & 0.762 & 0.053 \\
\hline 6 & $\# 1$ & 6.035 & 1.500 & 0.045 & 4.424 & 1.100 & 0.033 \\
\hline 7 & $\# 1$ & 4.726 & 1.131 & 0.056 & 3.009 & 0.702 & 0.035 \\
\hline 8 & $\# 1$ & 4.826 & 1.188 & 0.101 & 3.117 & 0.731 & 0.065 \\
\hline 9 & $\# 1$ & 5.023 & 1.167 & 0.083 & 3.330 & 0.788 & 0.055 \\
\hline 10 & $\# 1$ & 4.735 & 1.105 & 0.077 & 3.019 & 0.705 & 0.049 \\
\hline 11 & $\# 1$ & 5.049 & 1.196 & 0.153 & 3.359 & 0.796 & 0.102 \\
\hline 12 & $\# 1$ & 5.995 & 1.487 & 0.156 & 4.381 & 1.087 & 0.114 \\
\hline 13 & $\# 1$ & 4.853 & 1.139 & 0.186 & 3.146 & 0.738 & 0.121 \\
\hline 14 & $\# 2$ & 7.934 & 0.860 & 0.292 & 6.491 & 0.704 & 0.239 \\
\hline 15 & $\# 2$ & 8.213 & 0.904 & 0.360 & 6.792 & 0.748 & 0.298 \\
\hline 16 & $\# 2$ & 8.196 & 0.902 & 0.252 & 6.775 & 0.745 & 0.208 \\
\hline 17 & $\# 2$ & 8.309 & 0.920 & 0.047 & 6.896 & 0.763 & 0.039 \\
\hline 18 & $\# 2$ & 8.530 & 0.956 & 0.227 & 7.135 & 0.800 & 0.190 \\
\hline 19 & $\# 2$ & 8.180 & 0.899 & 0.355 & 6.757 & 0.743 & 0.293 \\
\hline 20 & $\# 2$ & 8.066 & 0.881 & 0.095 & 6.634 & 0.724 & 0.078 \\
\hline 21 & $\# 2$ & 8.196 & 0.902 & 0.230 & 6.775 & 0.745 & 0.190 \\
\hline 22 & $\# 3$ & 5.875 & 0.961 & 0.211 & 4.257 & 0.696 & 0.153 \\
\hline 23 & $\# 3$ & 5.998 & 0.987 & 0.073 & 4.389 & 0.722 & 0.053 \\
\hline 24 & $\# 3$ & 6.309 & 1.054 & 0.135 & 4.725 & 0.790 & 0.101 \\
\hline 25 & $\# 3$ & 6.438 & 1.083 & 0.048 & 4.864 & 0.818 & 0.036 \\
\hline 26 & $\# 3$ & 7.013 & 1.215 & 0.389 & 5.486 & 0.951 & 0.304 \\
\hline 27 & $\# 3$ & 7.013 & 1.215 & 0.112 & 5.486 & 0.951 & 0.087 \\
\hline 28 & $\# 3$ & 6.610 & 1.122 & 0.103 & 5.051 & 0.857 & 0.079 \\
\hline 29 & $\# 3$ & 5.924 & 0.971 & 0.618 & 4.310 & 0.706 & 0.449 \\
\hline 30 & $\# 3$ & 6.747 & 1.153 & 0.101 & 5.198 & 0.888 & 0.078 \\
\hline 31 & $\# 3$ & 6.022 & 0.992 & 0.045 & 4.415 & 0.727 & 0.033 \\
\hline 32 & $\# 3$ & 8.204 & 1.517 & 0.194 & 6.773 & 1.252 & 0.160 \\
\hline 33 & \#3 & 10.35 & 2.177 & 0.279 & 9.09 & 1.913 & 0.245 \\
\hline 34 & $\# 4$ & 5.403 & 1.444 & 0.252 & 2.914 & 0.779 & 0.136 \\
\hline 35 & $\# 4$ & 5.169 & 1.366 & 0.190 & 2.652 & 0.701 & 0.098 \\
\hline 36 & $\# 4$ & 5.326 & 1.418 & 0.238 & 2.828 & 0.753 & 0.127 \\
\hline 37 & $\# 4$ & 5.224 & 1.384 & 0.145 & 2.714 & 0.719 & 0.075 \\
\hline 38 & \#4 & 5.232 & 1.387 & 0.371 & 2.723 & 0.722 & 0.193 \\
\hline 39 & $\# 4$ & 5.380 & 1.436 & 0.460 & 2.888 & 0.771 & 0.247 \\
\hline 40 & $\# 4$ & 5.224 & 1.384 & 0.169 & 2.714 & 0.719 & 0.088 \\
\hline 41 & $\# 4$ & 5.411 & 1.447 & 0.296 & 2.923 & 0.782 & 0.160 \\
\hline 42 & $\# 4$ & 5.240 & 1.390 & 0.193 & 2.732 & 0.724 & 0.101 \\
\hline 43 & $\# 4$ & 4.405 & 1.165 & 0.149 & 2.661 & 0.704 & 0.090 \\
\hline 44 & $\# 4$ & 5.326 & 1.418 & 0.149 & 2.828 & 0.753 & 0.079 \\
\hline 45 & $\# 4$ & 5.341 & 1.423 & 0.198 & 2.845 & 0.758 & 0.106 \\
\hline 46 & $\# 4$ & 5.318 & 1.416 & 0.085 & 2.819 & 0.750 & 0.045 \\
\hline 47 & \#4 & 5.310 & 1.413 & 0.091 & 2.810 & 0.748 & 0.048 \\
\hline 48 & $\# 4$ & 5.310 & 1.413 & 0.197 & 2.810 & 0.748 & 0.104 \\
\hline
\end{tabular}


Table 3. Comparison between system reliability indices for nonautomated and automated distribution network

\begin{tabular}{|c|c|c|c|c|c|}
\hline $\begin{array}{c}\text { Operating } \\
\text { philosophy }\end{array}$ & Feeder & $\begin{array}{c}\text { SAIDI } \\
\text { (hr/yr) }\end{array}$ & $\begin{array}{c}\text { SAIFI } \\
\text { (int/yr) }\end{array}$ & $\begin{array}{c}\text { CAIDI } \\
\text { (hr/int) }\end{array}$ & $\begin{array}{c}\text { EENS } \\
\text { (MWh } \\
\text { r/yr) }\end{array}$ \\
\hline \multirow{4}{*}{$\begin{array}{c}\text { Non- } \\
\text { automated }\end{array}$} & Feeder 1 & 1.20 & 0.24 & 5.08 & 1.85 \\
\cline { 2 - 6 } & Feeder 2 & 0.91 & 0.11 & 8.23 & 1.86 \\
\cline { 2 - 6 } & Feeder 3 & 1.22 & 0.17 & 7.04 & 2.31 \\
\cline { 2 - 6 } & Feeder 4 & 1.40 & 0.26 & 5.37 & 3.18 \\
\cline { 2 - 6 } & System & 1.20 & 0.21 & 5.86 & 9.19 \\
\hline \multirow{4}{*}{ Automated } & Feeder 1 & 0.80 & 0.24 & 3.37 & 1.22 \\
\cline { 2 - 6 } & Feeder 2 & 0.75 & 0.11 & 6.81 & 1.53 \\
\cline { 2 - 6 } & Feeder 3 & 0.96 & 0.17 & 5.52 & 1.78 \\
\cline { 2 - 6 } & Feeder 4 & 0.75 & 0.26 & 2.86 & 1.7 \\
\cline { 2 - 6 } & System & 0.79 & 0.21 & 3.87 & 6.23 \\
\hline
\end{tabular}

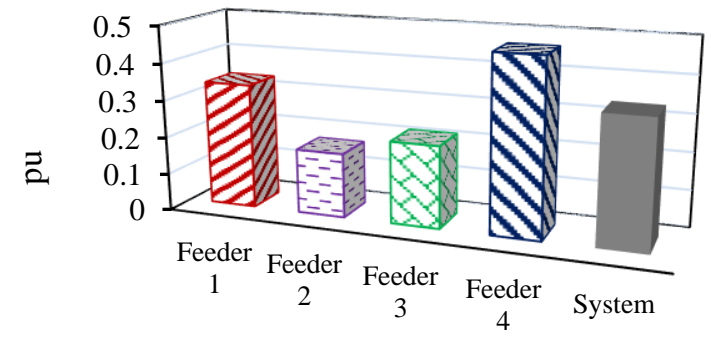

(a) Effect of distribution automation on SAIDI

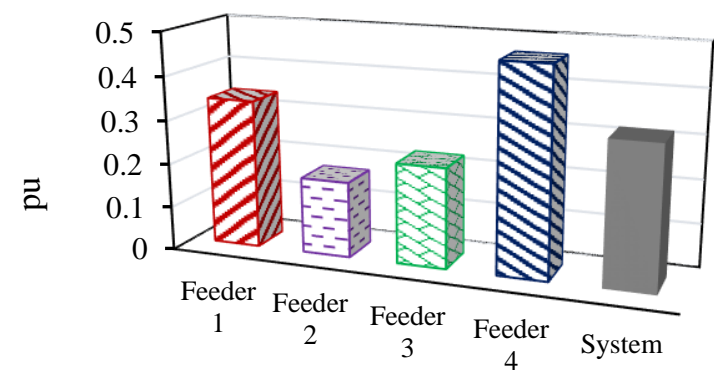

(b) Effect of distribution automation on EENS

Fig. 8. Reduction in SAIDI and EENS with ADAS

\section{CONCLUSIONS}

The growing importance of ADAS and its expected benefits on the operation of distribution systems encourage the investigation of this subject. The results show that the determination of the generic strategy for ADAS will avoid the huge investment toward a complete vision of smarter distribution systems. Following the proposed implemented ADAS strategy, FLISR application has been implemented on real, urban, underground $11 \mathrm{kV}$ distribution network based on local-centralized architecture. From the investigation, the presented architecture is defined as a cost-effective solution with the existing network. The results of reliability analysis for both automated and non-automated distribution network are evaluated and compared. It is concluded that the automated network with the proposed ADAS has a tangible benefit through considerable reduction in load point indices. Along with the same line, the system indices such as SAIDI and EENS exhibit a significant reduction by $34 \%$ and 32 $\%$, respectively. In addition, the proposed ADAS provides an intangible benefits observed by grater customer satisfaction and reduced penalties by industry regulators through the reduction of the outage time and outage cost.

\section{REFERENCES}

[1] Shang-Wen Luan; Jen-Hao Teng; Shun-Yu Chan; Lain-Chyr Hwang, "Development of an automatic reliability calculation system for advanced metering infrastructure," in Industrial Informatics (INDIN), 2010 8th IEEE International Conference on, pp. 342-347, 13-16 July 2010.

[2] M. McGranaghan and F. Goodman, "Technical and system requirements for Advanced Distribution Automation," Electricity Distribution, 2005. CIRED 2005. 18th International Conference and Exhibition on, Turin, Italy, 2005, pp. 1-5.

[3] R. W. Uluski, "The role of Advanced Distribution Automation in the Smart Grid," Power and Energy Society General Meeting, 2010 IEEE, Minneapolis, 2010, pp. 1-5.

[4] Shahin, M.A., "Smart Grid self-healing implementation for underground distribution networks," in Innovative Smart Grid Technologies - Asia (ISGT Asia), 2013 IEEE, pp.1-5, 10-13 Nov. 2013.

[5] Siirto, O., Kuru, J., and Lehtonen, M., "Fault location, isolation and restoration in a city distribution network," in Electric Power Quality and Supply Reliability Conference (PQ), 2014, pp. 367-370, 11-13 June 2014.

[6] Gomes, D.; Colunga, R.; Gupta, P.; Balasubramanian, A., "Distribution automation case study: Rapid fault detection, isolation, and power restoration for a reliable underground distribution system," in Protective Relay Engineers, 2015 68th Annual Conference on, 
pp.325-334, March 30 2015-April 22015.

[7] Kuru, J.; Ihonen, T.; Haikonen, J., "Controlcenter-based automatic fault isolation and restoration system for rural medium voltage networks," in Electricity Distribution (CIRED 2013), 22nd International Conference and Exhibition on , pp.1-4, 10-13 June 213.

[8] Chollot, Y.; Wild, J.; Berry, T.; Jourdan, A.; Raison, B.; Marguet, R.; Houssin, J.; Joubert, R., "Decentralized self healing solution tested in the framework of GreenLys smart grid project," in PowerTech 2013 IEEE, Grenoble, pp. 1-7, 16-20 June 2013.

[9] Coster, Edward; Kerstens, Wim; Berry, Tom, "Self healing distribution networks using smart controllers," in Electricity Distribution (CIRED 2013), 22nd International Conference and Exhibition on. Stockholm, pp.1-4, 10-13 June 2013.

[10] IEEE Distribution Automation Workig Group, "Smart Grid for Distribution Systems: The Benefits and Challenges of Distribution Automation(DA)," IEEE White Pap., 2007.

[11] Madani, V.; Das, R.; Aminifar, F.; McDonald, J.; Venkata, S.S.; Novosel, D.; Bose, A.; Shahidehpour, M., "Distribution Automation Strategies Challenges and Opportunities in a Changing Landscape," in Smart Grid, IEEE Transactions on , vol.6, no.4, pp.2157-2165, July 2015.

[12] R. Das, V. Madani, F. Aminifar, J. McDonald, S.S Venkata. D., Novosel, A.Bose and M. Shahidehpour, "Distribution Automation Strategies: Evolution of Technologies and the Business Case," inIEEE Transactions on Smart Grid, vol. 6, no. 4, pp. 2166-2175, July 2015.

[13] Späck H., Schüpferling B., Riemenschneider J. and Schelte M., "Intelligent transformer substations in modern medium voltage networks as part of "smart grid"," Power Generation Transmission, Distribution and Energy Conversion (MedPower 2010), 7th Mediterranean Conference and Exhibition on, Agia Napa, 2010, pp. 1-7.

[14] V. Gungor, D. Sahin, and T. Kocak, "Smart Grid Technologies: Communication Technologies and Standards," in IEEE Transactions on Industrial Informatics, vol. 7, no. 4, pp. 529-539, Nov. 2011.

[15] Yanfeng Gong; Guzman, A., "Integrated Fault Location System for Power Distribution Feeders," in Industry Applications, IEEE Transactions on , vol.49, no.3, pp.1071-1078, May-June 2013.

[16] Sudhakar, T. D., \& Srinivas, K. N. "Restoration of power network-a bibliographic survey," European Transactions on Electrical Power, vol. 21, no. 1, pp. 635-655, 2011.

[17] Subban, P.P., Awodele, K.O., "Reliability impact of different smart grid techniques on a power distribution system," in Innovative Smart Grid Technologies Latin America (ISGT LA), 2013 IEEE PES Conference On, pp.1-8, 15-17 April 2013.

[18] IEEE Guide for Electric Power Distribution Reliability Indices," in IEEE Std 1366-2003 (Revision of IEEE Std 1366-1998), pp.1-50, May 142004.

[19] IEEE Recommended Practice for the Design of Reliable Industrial and Commercial Power Systems," in IEEE Std 493-2007 (Revision of IEEE Std 493-1997), pp.1-689, June 252007.

[20] Ahmed R. Abul'Wafa, "Reliability Improvement of Radial Distribution System with Different Configurations of Disconnecting Switches," The 16th International Middle East Power Systems Conference (MEPCON'14). Cairo, Egypt, 3-25 December 2014. 